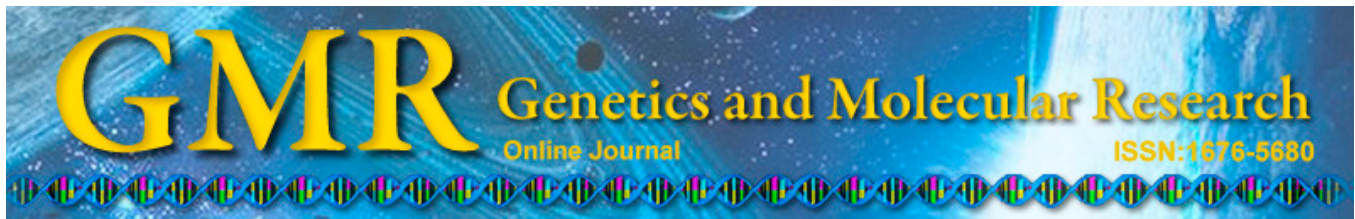

\title{
Molecular characterization and expression analysis of purple acid phosphatase gene from pearl oyster Pinctada martensii
}

\author{
Q.H. Wang, Y. Jiao, X.D. Du, X.X. Zhao, R.L. Huang, \\ Y.W. Deng and F. Yan \\ Fishery College, Guangdong Ocean University, Zhanjiang City, \\ Guangdong, China \\ Corresponding author: X.D. Du \\ E-mail: gdhddxd@hotmail.com
}

Genet. Mol. Res. 14 (1): 552-562 (2015)

Received January 24, 2014

Accepted May 15, 2014

Published January 26, 2015

DOI http://dx.doi.org/10.4238/2015.January.26.10

\begin{abstract}
Purple acid phosphatases (PAPs), also known as type 5 acid phosphatases, are widely present in animals, plants, and fungi. In mammal, PAP was reported to participate in immune defense and bone resorption. In this study, the characteristics and potential functions of a PAP gene from pearl oyster Pinctada martensii (pm-PAP) were examined. The Pm-PAP cDNA was found to be 2777 base pairs, containing a 1581base pair open reading fragment encoding for 526 amino acids with an estimated molecular mass of $60.1 \mathrm{kDa}$ and theoretical isoelectric point

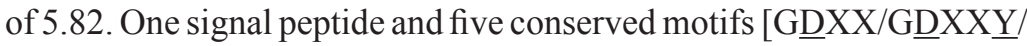
$\mathrm{GNH}(\mathrm{D} / \mathrm{E}) / \mathrm{XXX} \underline{\mathrm{H}} /(\mathrm{A} / \mathrm{G}) \underline{\mathrm{H}} \underline{\mathrm{H}}]$ were present in the entire sequence. Tissue expression profile analysis showed that pm-PAP mRNA was constitutively expressed in all tissues studied with abundant mRNA found in mollusk defense system, including hepatopancreas, gill, and hemocytes. After lipopolysaccharide stimulation, the expression of pm-PAP mRNA in hemocytes was dramatically upregulated at $2 \mathrm{~h}$ and achieved the highest level at $36 \mathrm{~h}$. Additionally, pm-PAP mRNA expression was significantly increased and achieved the highest level
\end{abstract}


at 2 days after the surgical implantation during pearl production. These results suggest that pm-PAP is a constitutive and inducible protein that may be involved in the immune defense of pearl oyster.

Key words: Immune response; Pearl oyster; Pinctada martensii; Purple acid phosphatase

\section{INTRODUCTION}

Purple acid phosphatases (PAPs) are a class of metalloenzymes that contain a binuclear metal ion complex in the active site (Flanagan et al., 2006). Because PAPs are insensitive to tartrate inhibition, these metalloenzymes are also known as tartrate-resistant acid phosphatases (Fleckenstein and Drexler., 1997; Oddie et al., 2000). PAPs are distinguished from other phosphatases based on their acidic $\mathrm{pH}$ optima and characteristic purple appearance resulting from a tyrosinate Fe(III) charge transfer (Schenk, 2013). PAPs are widely distributed in eukaryotes, including in animals, plants, and fungi. All members of the PAP family con-

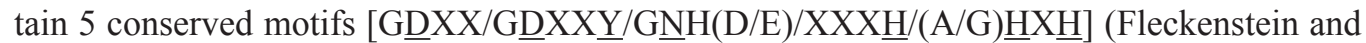
Drexler, 1997; Flanagan et al., 2006; Dionisio et al., 2011). The 7 underlined invariant amino acid residues distributed in these 5 conserved motifs are involved in the coordination of the dimetallic center in the active site (Flanagan et al., 2006).

In mammals, PAPs are involved in the immune response (Brehme et al., 1999; Bune et al., 2001; Hayman et al., 2001; Räisänen et al., 2005). This report is supported by the finding that PAPs co-localize with alpha2-macroglobulin $\left(\alpha_{2} M\right)$, and its expression can be upregulated after lipopolysaccharide (LPS) stimulation (Brehme et al., 1999; Hayman et al., 2001). Overexpression of PAPs in a macrophage-like cell line resulted in increased reactive oxygen species (ROS) production and an enhanced capacity for bacterial killing (Halleen et al., 2003; Räisänen et al., 2005). Furthermore, PAP knockout mice showed a reduced capacity to clear bacterial pathogens from the peritoneal cavity in vivo (Bune et al., 2001). In osteoclasts, PAPs may participate in bone resorption and are used as histochemical markers (Hayman et al., 1996; Feder et al., 2012).

Pearl oyster Pinctada martensii is an important species cultured for marine pearl production in China and Japan (Jiao et al., 2012). In pearl production using cultured pearl oysters, a mantle graft and a nucleus are implanted into the gonad of a host pearl oyster; this surgical implantation process can induce the immune response of the host pearl oyster ( $\mathrm{Li}$ et al., 2010). To elucidate the mechanism of this immune response, various immune-related genes such as LPS-induced tumor necrosis factor- $\alpha$ factor (Zhang et al., 2009a), inhibitor of nuclear factor-kappaB (IkappaB) (Zhang et al., 2009b), inhibitor of nuclear factor-kappaB kinase homolog (Xiong et al., 2008), and F-type lectin (Chen et al., 2011) have been cloned and functionally examined. Although some PAP genes have been identified in mollusks through sequence analysis, the PAP gene has not been characterized in detail and identified in P. martensii. To understand the immune response in mollusks, we cloned the full-length cDNA of a PAP gene from P. martensii (pm-PAP). We also analyzed the distribution of pmPAP mRNA in adult tissues and temporal expression patterns after a challenge with LPS. Furthermore, we compared changes in pm-PAP expression after surgical implantation during pearl production. 


\section{MATERIAL AND METHODS}

\section{Pearl oyster and immune challenge}

Host oysters (approximately 2 years of age) were pre-conditioned for 2 weeks prior to nucleus implantation. Mantle grafts were randomly cut from the donor oysters (shell length of 60-65 mm; approximately 1.5 years of age). At $0,1,2,3,5,10,15$, and 20 days after surgical implantation, hemocytes in the host pearl oysters were collected, immersed in Trizol (Invitrogen, Carlsbad, CA, USA), and stored in liquid nitrogen.

For the immune challenge, pearl oysters (approximately 2 years of age) were obtained from Liushagang (Zhanjiang, Guangdong Province, China). They were maintained at $25^{\circ}-27^{\circ} \mathrm{C}$ in tanks with recirculating seawater for 1 week before the experiment. Next, 100 $\mu \mathrm{L}$ phosphate-buffered saline and $100 \mu \mathrm{L} 10 \mu \mathrm{g} / \mathrm{mL}$ LPS (Sigma, St. Louis, MO, USA) were injected into the adductor muscle of the pearl oysters in the control group and in the challenged group, respectively. Hemocytes were collected from 6 pearl oysters at 0, 2, 4, 8, 12, 24, and 36 $\mathrm{h}$ post-injection, immersed in Trizol, and stored in liquid nitrogen.

\section{Rapid amplification of cDNA ends (RACE)}

A SMART RACE cDNA amplification kit (Clontech, Mountain View, CA, USA) was used for 5'/3' RACE to obtain the full-length cDNA of pm-PAP. Total RNA used for the RACE reaction was extracted from the hemocytes of $P$. martensii using Trizol. Gene-specific primers were designed based on the amino acid sequences of the PAP cDNA fragment obtained from the transcriptome of P. martensii (Zhao et al., 2012). Table 1 shows the inner and the outer polymerase chain reaction (PCR) primers.

\begin{tabular}{lll}
\multicolumn{2}{c}{ Table 1. Primers for gene amplification and characterization. } & \\
\hline Primer & Sequence (5'-3') & Application \\
beta-actin-F & 5'-TCCCTGGAGAAGAGC-3' & qRT-PCR \\
beta-actin-R & 5'-AGTTTCGTGGATGCC-3' & qRT-PCR \\
3'RACE-PAP1 & 5'- CTGTGAAACCGAGGGCAGAACTTGA-3' \\
3'RACE-PAP2 & 5'-CAGGTGCCCGTTCCATTCCATTAC-3' \\
5'RACE-PAP1 & 5'-ACAGAACTGTTACTGCGGGAACCAA-3' \\
5'RACE-PAP2 & 5'-TAATGTCGTCAGAGTTCCCCAGGCT-3' & Outer PCR \\
PAP-F & 5'-AGTGCCTACAGTAATCTCG-3' & Inner PCR \\
PAP-R & 5'-TCTGCTGGTACAATTCATC-3' & Outer PCR \\
\hline
\end{tabular}

\section{DNA sequencing and bioinformatic analysis}

The PCR products of the $5^{\prime}$ and $3^{\prime}$ ends were purified, sub-cloned into the pGEM-T easy vector (Promega, Madison, WI, USA), and sequenced. All cDNA sequences were analyzed using the BLAST program available from the National Center for Biotechnology Information (http://www.ncbi.nlm.nih.gov/). The open reading fragment (ORF) was characterized using the GENSCAN (http://genes.mit.edu/GENSCAN.html) and the ORF Finder (http:// www.ncbi.nlm.nih.gov/gorf/orfig.cgi). The primary structure, including amino acid composition, molecular weight, and theoretical isoelectric point, was analyzed using the protparam tool (http://web.expasy.org/cgi-bin/protparam/protparam). The SignalP-4.0 (http://www.cbs. 
dtu.dk/services/SignalP/) was used to analyze the signal peptide. The MEGA 4.0 software program was used to construct a phylogenetic tree.

\section{Tissue-specific expression and temporal expression of pm-PAP after LPS stimulation or surgical implantation}

The adductor muscle, gill, pearl sac, mantle, hepatopancreas, gonad, foot, and hemocytes were collected from the pearl oysters and immediately stored in liquid nitrogen until use. Total RNA was isolated using Trizol reagent according to manufacturer instructions. RNA integrity was determined by electrophoresis on a $1.2 \%$ agarose gel. RNA concentration and the purity were examined using a NanoDrop ND1000 spectrophotometer (Wilmington, DE, USA) at 260 and $280 \mathrm{~nm}$, respectively. Total RNA in adult tissues from the pearl oyster was reverse transcribed using oligo (dT)16 and M-MLV reverse transcriptase (Clontech) to produce cDNA according to manufacturer instructions. pm-PAP mRNA expression was determined by quantitative real-time RT-PCR (qRT-PCR) with $\beta$-actin as an internal control (Jiao et al., 2012). Table 1 shows the gene-specific primer sets with the resulting product size of 112 base pairs (bp). Amplifications were conducted using SYBR green according to the manufacturer protocol. Fluorescent real-time PCR was performed in a C1000TM thermal cycler CFX96TM real-time system (Bio-Rad, Hercules, CA, USA). Data were analyzed using the Bio-Rad CFX manager software.

\section{Statistical analysis}

The data from the experiments were analyzed using SPSS 13.0 (SPSS, Inc., Chicago, IL, USA). A P value $<0.05$ was considered to be statistically significant.

\section{RESULTS}

\section{cDNA cloning, sequencing, and characterization of the pm-PAP gene}

By using the unigene sequence, which was annotated to PAP from the P. martensii transcriptome database, we designed 4 gene-specific primers that were used to amplify the $5^{\prime}$ and 3' nucleotide sequences using 3'- and 5'-RACE technology. The PCR products were cloned and sequenced. Figure 1 shows that the complete sequence of pm-PAP cDNA contained a $5^{\prime}$ terminal untranslated region of $44 \mathrm{bp}$, an ORF of $1581 \mathrm{bp}$, and a 3' untranslated region of 1152 bp with a 28 bp poly(A) tail. The pm-PAP sequence was deposited in the GenBank with Accession No. KC888746.

\section{Characterization of the deduced amino acid sequence of the pm-PAP gene}

The deduced protein pm-PAPs encoded 526 amino acids; the protein had a mature molecular weight of $60.1 \mathrm{kDa}$ and a theoretical isoelectric point of 5.82. The amino acid se-

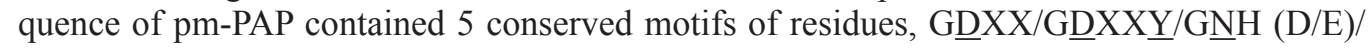
$\mathrm{XXX} \underline{H} /(\mathrm{A} / \mathrm{G}) \underline{\mathrm{H} X} \underline{\mathrm{H}}$ (black box), which are commonly found in PAPs in other species (Flanagan et al., 2006). A signal peptide was predicted in the first 18 amino acids. Two glycosylation sites were found at positions 63 and 120 of this peptide (Figure 1). The deduced protein se- 
quence of pm-PAP was multialigned with other known PAPs. The results showed that pm-PAP had significant homology to a variety of other known PAPs (Figure 2).

1 acatggggacatacaggctgacctaggattatacggacaaagaa 45 atgggttggattagcttccttattctgggctttcagctctagtcaggggtctggaccgcccagaacagatccatctcagcctggggaac $M G W I S F L I L G F S A L V R G L D R P$ I $\quad$ I I H L S L G N 135 tctgacgacattatgacggtaatgtgggctacatccgatgacgtcactggtcacgtgatgtatggagaatcgattaacaaccttggttcc $S$ D $D$ I $M$ T V M W A T S D D V T G H V M Y G E S I N N L G S 225 cgcagtaacagttctgttgccactttacaaactgatagctggaacgctatgaagaatatccatagagcacaaatgaatgggctggtgtcc R S N S S V A T L Q T D S W N A M K N I H R A Q $M$ 315 ggatctacttacttttacaaggtggtattaagttccggagaggagaaggttgagagctctacatacagctttacaacaatatcacaaaac $G S T Y F$ Y V V L S S G E E K V E S S T Y S F T T I S Q 405 aagacaaagccccgtaaattccttgtttacggtgacctcggaaaagtggagggaaaacctacgtttcctgtttaaaggaggaagtggac K T K P R K F L V Y G D L G K V E G K P T F P V L K E E V D

495 agcggtgaatatgacgtcatatggcacgtcggggattttgcctataatatggaatctgacggcggaaagacaggtgatgacttttatca

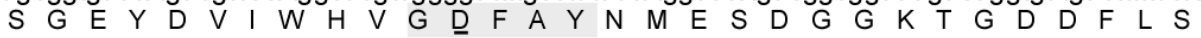
585 gaaattgaaccaatcgcttcacggataccttacatgacggcacctggaaatcatgaactgggcaatcagctacatcattacagaacacgg

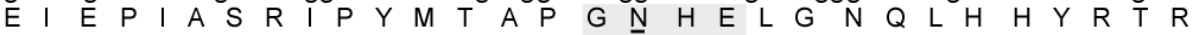
675 ttctcgatgccaggaacaacatggccgatgtcagaagacagactgtggtacagttacaatattggtctggtccatttataagctactcc F S M P G T T W P M S E D R L W Y S Y N I G L V H F I S Y S 765 acagaagtgtactttatcgacaatcaggactatgtctgtcagcaatattattggctactacaggatctaataacagccaatcagaatcga

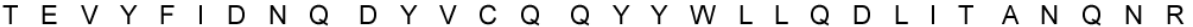
855 gataagcagccatggattgtggcaatgggtcatcgacctatgtattgctccaataaggacgccgacgactgtactggacgtatatttggc $\begin{array}{llllllllllllllllllllllllllllll}D & K & Q & P & W & \text { I } & V & A & M & G & \underline{H} & R & P & M & Y & C & S & N & K & D & A & D & D & C & T & G & R & I & F & G\end{array}$ 945 tactgggtaaaacatgggttagaggatcttttcaaggccagggagttgactttgtaatccaagctcacgagcactcgtatgaacgcctt $Y W V K$ H G L E D L F Q G Q G V D F V I Q A 1035 tggcccatgtacaatgagaaaattatcgccaaaaactacgtggacccggaagcacttgttcacgtgacttccggtgcagcaggctgtgga W $P$ M Y N E K I I A K N Y V D P E A L V H V T $S$ G A A G C G 1125 gaaatagtagacaaaatgggagaaccagatccatggtcggcatttcgtgcagatacaaaaagtttcattctttggaaagctcatagta

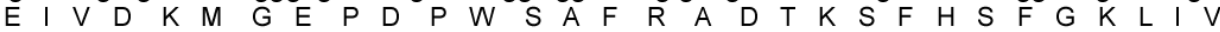
1215 tacaataatactcatttacaatttatacaagaaactgtgaaaccgagggcagaacttgacaatttctggatcgtacaacacaatcacggt $\begin{array}{llllllllllllllllllllllllllll} & N & N & T & H & L & Q & F & Q & Q & E & T & V & K & P & R & A & E & L & D & N & F & W & V & Q & H & N & H\end{array}$ 1305 ccacgcttcgataacgtcgactgtcaaaacaaatctcacgcaaattcaatctgcaggtgcccgtttccattccattacgtcacagtggct $\begin{array}{lllllllllllllllllllllllllll}P & R & F & D & V & D & C & Q & N & K & S & H & A & N & S & I & C & R & C & P & F & P & F & H & Y & V & T\end{array}$ 1395 gttgcgtcaggaataacacttgttctcgtcatcgtgatggcttgtatttggtgctgccgtcgatgcggactatgtcattgttcaaggaca

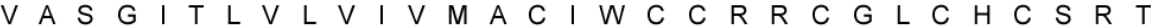
1485 acctgttcatgttgtaggaaacagtccgtctggacgaaaatgaggatgaaatttgaaaagtcttctagagacaaaagctacgtgcatccg $T$ C S C C R K Q S V W T K M R M K F E K S S R D K S Y V V H $P$ 1575 atagactgtgatacgtataatctattagacaacgaagacgtagaaatttgatgagaaatcttcaatcttttaatgaaaaatcatgtgcc I D C D T Y N L L D N E D V E I *

1665 aaatactataccgaaatgtcccgataaatattttatttagatttatattttacaataattttaaactattttaaatccatcattggtgc 1755 tttagatctgcatgatttaatatattaccacaagagtattctagacataaatacgaaaataatacttttaaaatattttgttgaataag 1845 ctttctgaatttattacattaacatgtgacattcctacgaaatgtgcgcgacgttacgtcacgtcaaaatgctttgacattatccatatt 1935 gcagaccgttcgggtgtcatgttccccctaggctacaaattctgaaatcatcaatgtctatacaatgttcatgttcgcccgagggtcggg 2025 attagttaatgacccaatttttcttggtacccgattcgactactagtatatttacataagatttgcacttagtcatgcgtatttctgtc 2115 cagtacctttctggaaaaaaatccaaaatattcaatattaactcaaacaaatg ttatttctgagatgattttggcagcttgtatttattt 2205 gtatacaatgtacgtacatctacaaggaaatttttacttgtttgttgtttatgtcgtatgtatattatgaaaaaacttaatacaataca 2295 tttacaagtacaatgtatgataacatgtagaccaaagacacggtgggaatgcatttttctgcaactcattgataatatcaattaaactt 2385 cgtgtggatagtggatatatccacatgtatgcgtttcaaatag cttgagcaaaaagttacaagattcaagatttaatatttatttctaac 2475 ataatcagaaatcataccatgcttttccactttctttatattcttcaatcattccatgattatatcgatatcatcttcatatatacac 2565 gtatattataactattacttacaatttgatcatatttatactgactcgttcatgaattacttttaaaattcatgaaatcgaaaatcacac 2655 atgacctagcttgaggcattttactaacataatgataaatcagacgattctactatgcattgttacatatacatgtatacgtttttaca 2745 aaaataaaaaaaaaaaaaaaaaaaaaaaaaaa

Figure 1. Nucleotide and amino acid sequences of pm-PAP. The numbers on the left indicate the positions of the nucleotides in the pm-PAP cDNA sequence. The putative signal peptide (residues 1-18) is italicized and the putative

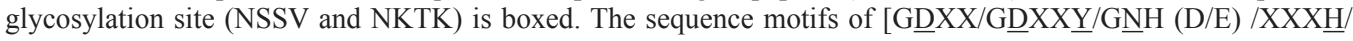
$(\mathrm{A} / \mathrm{G}) \underline{\mathrm{HX}} \underline{\mathrm{H}}]$ are shaded. 


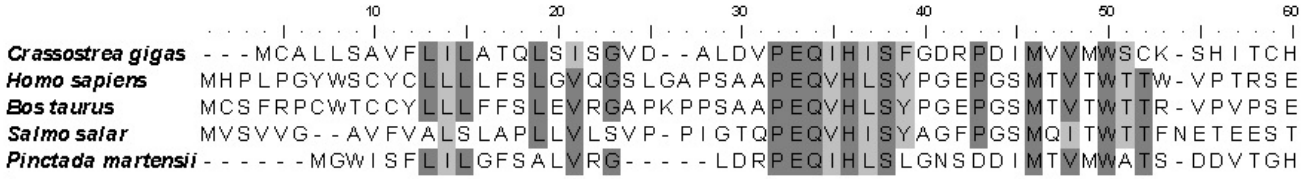

\begin{tabular}{ll|lll} 
& 10 & 10 & 100 & 110
\end{tabular}

$\begin{array}{llll} & & 100 & 110\end{array}$

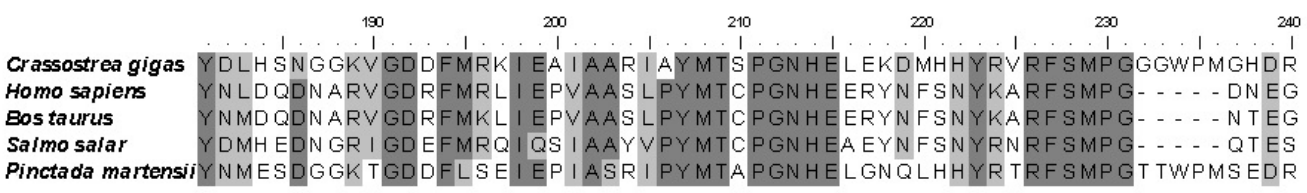
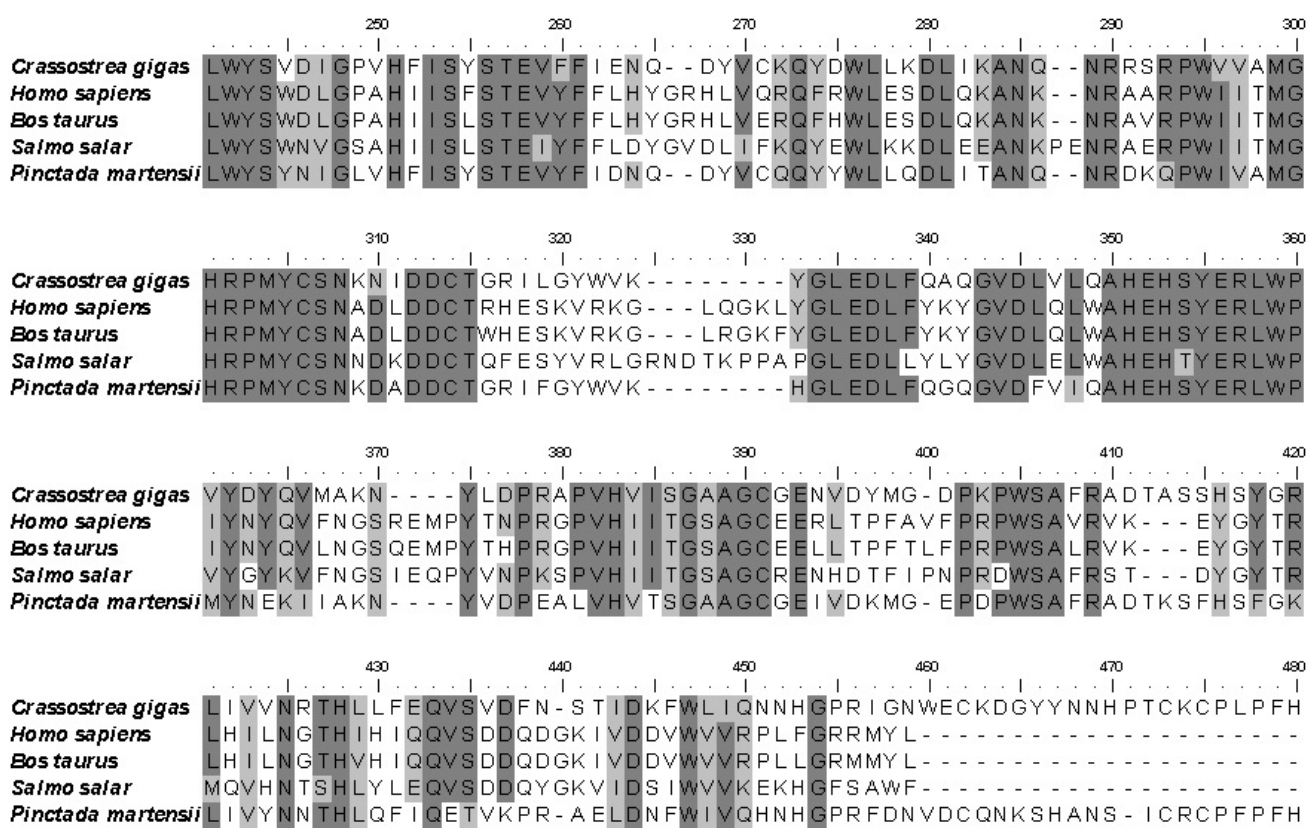

Figure 2. Alignment of full-length amino acid sequence of pm-PAP with other known PAP sequences. Identical amino acids are highlighted in dark gray and similar amino acids are highlighted in light gray. The origin for each PAP sequence isindicated on the left. 


\section{Quantitative analysis of pm-PAP gene expression in different tissues}

qRT-PCR analysis was performed to determine the tissue-specific expression of pmPAP mRNA with $\beta$-actin as an internal control. The results showed that pm-PAP mRNA was constitutively expressed in all examined tissues (adductor muscle, gill, pearl sac, mantle, hepatopancreas, gonad, foot, and hemocytes). Figure 3 shows that pm-PAP mRNA was highly expressed in the hepatopancreas, gill, and hemocytes, which are involved in the defense system of mollusks. These results suggest that pm-PAP is involved in the immune response of $P$. martensii.

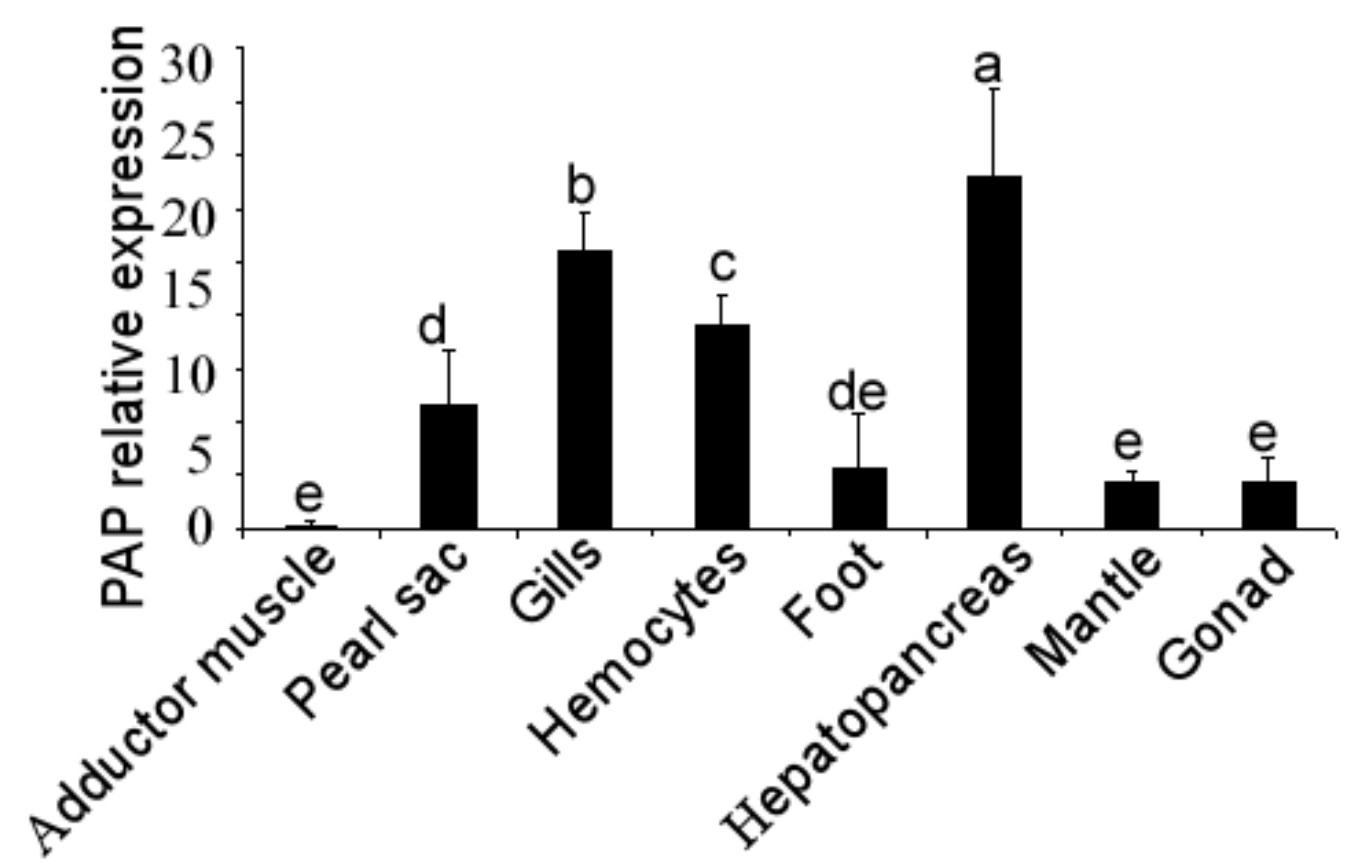

Figure 3. pm-PAP mRNA expression pattern in different tissues. qRT-PCR was performed using RNA samples from the adductor muscle, gill, pearl sac, mantle, hepatopancreas, gonad, foot, and hemocytes. The $\beta$-actin gene of the pearl oyster was used as an internal control.

\section{Temporal expression pattern of pm-PAP after LPS stimulation}

To validate the function of pm-PAP in the immune response, we analyzed the temporal expression of the pm-PAP gene in hemocytes after LPS stimulation by qRT-PCR. The expression level of pm-PAP was significantly increased at $2 \mathrm{~h}$ and maintained at a relatively stable level until $12 \mathrm{~h}$. pm-PAP mRNA expression was upregulated again at 24 $\mathrm{h}$ after LPS stimulation. The expression level was 7.24-fold higher than that in the control group at $36 \mathrm{~h}$ (Figure $4 \mathrm{~A})$. 


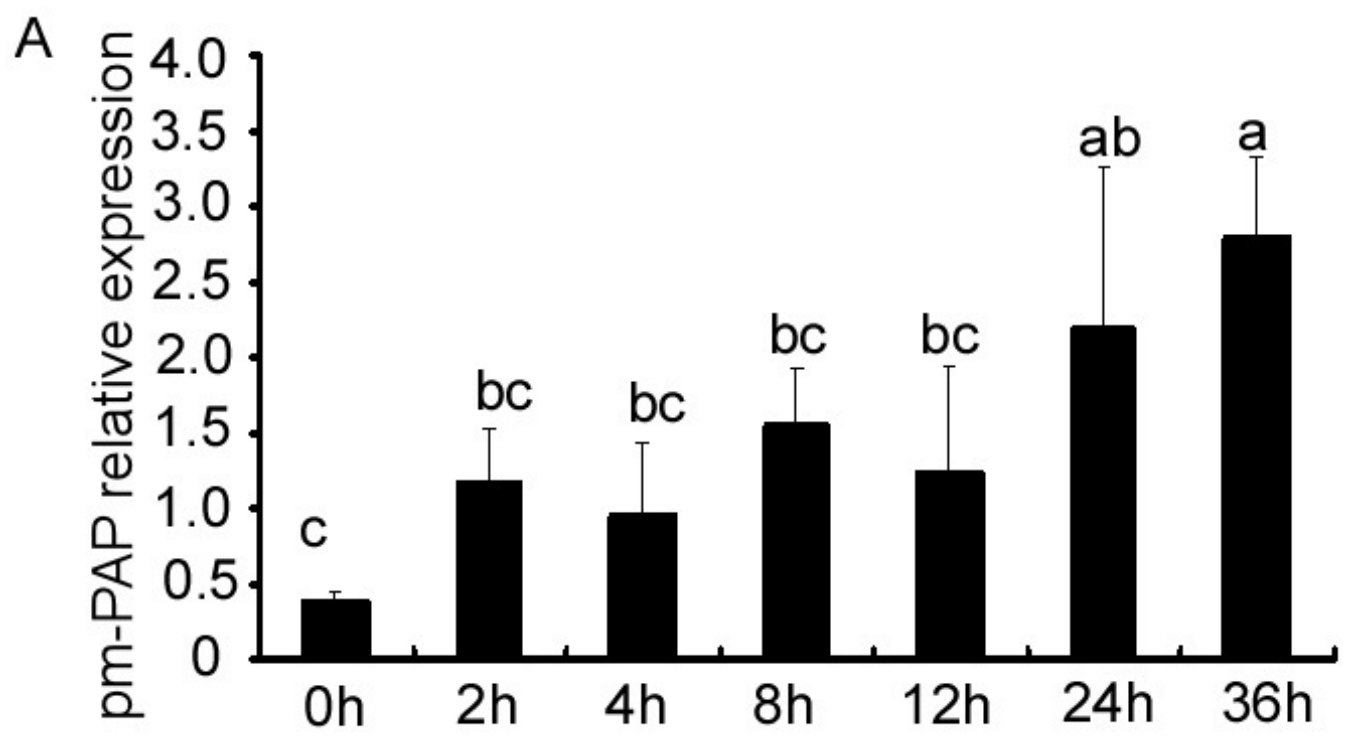

B

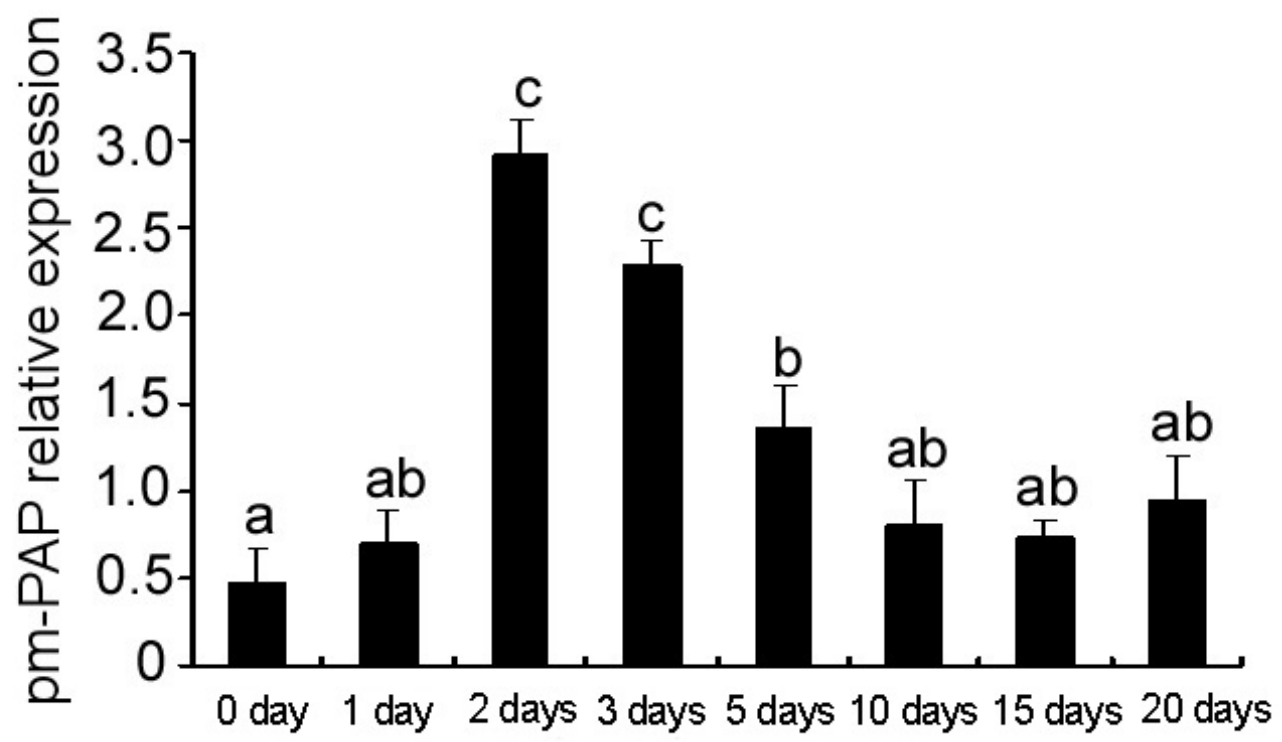

Figure 4. Expression level of pm-PAP mRNA after LPS stimulation and surgical implantation. (A) qRT-PCR was performed using the RNA samples from hemocytes at $0,2,4,8,12,24$, and $36 \mathrm{~h}$ after LPS stimulation. (B) qRTPCR was performed using the RNA samples from hemocytes at $0,1,2,3,5,10,15$, and 20 days after surgical implantation. The $\beta$-actin gene of the pearl oyster was used as an internal control.

\section{Temporal expression pattern of pm-PAP after surgical implantation}

Surgical implantation in pearl production can induce an immune response in the host pearl oyster. To further validate the function of pm-PAP in the immune response, we detected 
the expression patterns of pm-PAP after surgical implantation. pm-PAP mRNA expression was significantly upregulated and reached its highest level at 2 days. Expression was gradually downregulated and then returned to the level of the control group at 10 days after surgical implantation, indicating an enhanced immune response after surgical implantation (Figure 4B).

\section{DISCUSSION}

In mammals, 2 types of PAPs have been identified: high-molecular weight ( $\sim 55$ $\mathrm{kDa})$ and low-molecular weight $(\sim 35 \mathrm{kDa})$. The pm-PAP obtained was 526 amino acids and had a mature molecular weight of $60.1 \mathrm{kDa}$. In mollusks, 3 PAP genes were obtained from C. gigas based on genome sequence analysis. All of these PAP genes contained a mature molecular weight of $\sim 60 \mathrm{kDa}$. In the recent transcriptome database of P. martensii (Zhao et al., 2012), a PAP gene with a lower molecular weight has not been identified. This result may be attributed to PAP genes in mollusks. Similarly to PAP genes from other species, the

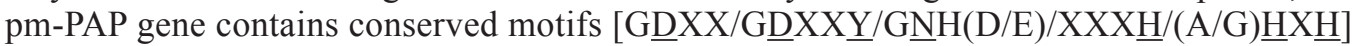
(Flanagan et al., 2006), coordinating the 2 metal ions using the 7 conserved amino acids underlined in the conserved motif. However, the metal ion composition of pm-PAP remains unknown. Because pm-PAP is homologous to other PAPs, Fe(III) may be 1 of the metal ions. The second metal ion may be Fe(II), $\mathrm{Zn}$ (II), or Mn(II). These metals constitute the redox-active center of PAPs. Two glycosylation sites were identified at positions 63 and 120 of the peptide. Glycosylation is a distinct feature of PAPs. Particularly, 5-10\% of the PAP molecular mass is carbohydrate content. Additionally, N-glycosylation may influence the catalytic activity of this enzyme (Wang et al., 2005).

Mammalian PAPs, abundantly expressed in macrophages, dendritic cells, and osteoclasts, with an important biological functions in the immune defense system and in bone resorption (Hayman et al., 1996, 2001; Räisänen et al., 2005). To validate the function of pm-PAP, we detected pm-PAP mRNA expression patterns in different tissues of P. martensii. Interestingly, pm-PAP was largely expressed in the hepatopancreas, gill, and hemocytes. The hepatopancreas of mollusks is regarded as an important immune organ that can secrete various enzymes to hydrolyze microorganisms and participate in digestive and defense functions (Tiscar and Mosca., 2004). The molluscan gill, which is the main interface between aquatic organisms and the external environment, is considered to be the first line of defense against bacterial infection (Chen et al., 2011; Lee et al., 2012). In invertebrates, hemocytes function as phagocytes are involved in various host immune responses such as recognition, phagocytosis, encapsulation, and oxidative killing; the hemocytes contain many types of immune factors and endogenous enzymes (Rebelo et al., 2013). This high expression of pm-PAP mRNA in these defense tissues suggest that pm-PAP may have specific functions in immune defense.

We detected pm-PAP expression patterns after LPS stimulation to validate the function of pm-PAP in the immune response. LPS are large molecules consisting of a lipid and a polysaccharide. These molecules are found in the outer membrane of Gram-negative bacteria and function as endotoxins that elicit a strong immune response in animals (Kilár et al., 2012). PAP expression is significantly upregulated at $48 \mathrm{~h}$ after LPS stimulation in human macrophages (Bune et al., 2001). However, the mechanism regulating PAP expression is not wellunderstood. Because PAP catalyzes the formation of ROS through Fenton's reaction (Halleen et al., 2003), upregulation of PAP expression can increase ROS production and enhance bacte- 
rial killing in host organisms (Räisänen et al., 2005). In P. martensii, pm-PAP expression was also upregulated at 2 and $24 \mathrm{~h}$ after LPS stimulation. The first upregulation at $2 \mathrm{~h}$ observed in our study may be induced directly by an existing cellular transcription factor. The second upregulation at $24 \mathrm{~h}$ may be induced by the newly synthesized transcription factor after LPS stimulation. Thus, pm-PAP observed in this study may function in the immune response similarly to the mechanism in mammalian PAP genes.

The immune rejection response may be one of the most important reasons for the induced nucleus rejection after surgical implantation during pearl production. The nucleus and the mantle graft, which were delivered into the host pearl oyster by surgical implantation, were recognized as foreign objects by the host pearl oyster. During surgical implantation, some microorganisms are transported into the body of the host pearl oyster (Li et al., 2010). Thus, surgical implantation may induce the immune response of the host pearl oyster, and a very strong immune response may lead to nucleus rejection or death of the host pearl oyster. The immune system of the host pearl oyster may also affect pearl sac development. The mechanism of the immune response must be understood in order to determine the mechanism of pearl sac development. This information will help to improve the quality and quantity of pearls and this information can be applied in pearl production. The immune response of the host pearl oyster after surgical implantation has attracted considerable attention. For instance, $\mathrm{Li}$ (2010) reported that some immune factors such as $\alpha_{2} \mathrm{M}$, acid phosphatase, and superoxide dismutase are significantly increased after surgical implantation. This result indicates that the stress caused by surgical implantation activates the defense system of hemocytes and subsequently changes the levels of various endogenous enzymes. In this study, pm-PAP was significantly upregulated at 2 days after surgical implantation. As reported in mammals, PAP can increase ROS production (Halleen et al., 2003), which is highly toxic to bacteria. Therefore, the increase in PAP expression after surgical implantation in the host pearl oyster may help in the resistance to bacterial infection.

In conclusion, using the partial sequence obtained from the transcriptome of the pearl sac, we obtained full-length pm-PAP cDNA from $P$. martensii and then analyzed the characteristics of the ORF and peptide sequence. pm-PAP mRNA was highly expressed in molluscan defense tissues, including the hepatopancreas, gill, and hemocytes. After LPS stimulation or surgical implantation, expression of pm-PAP mRNA was significantly upregulated, suggesting that pm-PAP functions in immune defense pathways of pearl oysters. Further studies examining the function of pm-PAP in nacre formation are necessary as mammalian PAPs participate in bone resorption.

\section{ACKNOWLEDGMENTS}

Research supported by the National Natural Science Foundation of China (\#31272635, \#41206141, \#31372526, \#31201023), Guangdong Natural Science Foundation (\#S2012040008042), Guangdong Province breeding project fund (\#2012LYM 0074), and Natural Foundation of Guangdong Ocean University (\#1212318).

\section{REFERENCES}

Brehme CS, Roman S, Shaffer J and Wolfert R (1999). Tartrate-resistant acid phosphatase forms complexes with alpha2macroglobulin in serum. J. Bone Miner. Res. 14: 311-318.

Bune AJ, Hayman AR, Evans MJ and Cox TM (2001). Mice lacking tartrate-resistant acid phosphatase (Acp 5) have 
disordered macrophage inflammatory responses and reduced clearance of the pathogen, Staphylococcus aureus. Immunology 102: 103-113.

Chen J, Xiao S and Yu Z (2011). F-type lectin involved in defense against bacterial infection in the pearl oyster (Pinctada martensii). Fish Shellfish Immunol. 30: 750-754.

Dionisio G, Madsen CK, Holm PB, Welinder KG, et al. (2011). Cloning and characterization of purple acid phosphatase phytases from wheat, barley, maize, and rice. Plant Physiol. 156: 1087-1100.

Feder D, Hussein WM, Clayton DJ, Kan MW, et al. (2012). Identification of purple acid phosphatase inhibitors by fragment-based screening: Promising new leads for osteoporosis therapeutics. Chem. Biol. Drug Des. 80: 665-674.

Flanagan JU, Cassady AI, Schenk G, Guddat LW, et al. (2006). Identification and molecular modeling of a novel, plantlike, human purple acid phosphatase. Gene 377: 12-20.

Fleckenstein E and Drexler HG (1997). Tartrate-resistant acid phosphatase: gene structure and function. Leukemia 11: $10-13$.

Halleen JM, Räisänen SR, Alatalo SL and Väänänen HK (2003). Potential function for the ROS-generating activity of TRACP. J. Bone Miner. Res. 18: 1908-1911.

Hayman AR, Jones SJ, Boyde A, Foster D, et al. (1996). Mice lacking tartrate-resistant acid phosphatase (Acp 5) have disrupted endochondral ossification and mild osteopetrosis. Development 122: 3151-3162.

Hayman AR, Macary P, Lehner PJ and Cox TM (2001). Tartrate-resistant acid phosphatase (Acp 5): identification in diverse human tissues and dendritic cells. J. Histochem. Cytochem. 49: 675-684.

Jiao Y, Wang H, Du X, Zhao X, et al. (2012). Dermatopontin, a shell matrix protein gene from pearl oyster Pinctada martensii, participates in nacre formation. Biochem. Biophys. Res. Commun. 425: 679-683.

Kilár A, Dornyei Á and Kocsis B (2012). Structural characterization of bacterial lipopolysaccharides with mass spectrometry and on- and off-line separation techniques. Mass Spectrom. Rev. 32: 90-117.

Lee Y, Wickamarachchi WD, Whang I, Oh M, et al. (2012). Immune response-related gene expression profile of a novel molluscan IкB protein member from Manila clam (Ruditapes philippinarum). Mol. Biol. Rep. 40: 1519-1527.

Li W, Shi Z and He X (2010). Study on immune regulation in Hyriopsis cumingii Lea: effect of pearl-nucleus insertion in the visceral mass on immune factors present in the hemolymph. Fish Shellfish Immunol. 28: 789-794.

Oddie GW, Schenk G, Angel NZ, Walsh N, et al. (2000). Structure, function, and regulation of tartrate-resistant acid phosphatase. Bone 27: 575-584.

Räisänen SR, Alatalo SL, Ylipahkala H, Halleen JM, et al. (2005). Macrophages overexpressing tartrate-resistant acid phosphatase show altered profile of free radical production and enhanced capacity of bacterial killing. Biochem. Biophys. Res. Commun. 331: 120-126.

Rebelo M de F, Figueiredo Ede S, Mariante RM, Nobrega A, et al. (2013). New insights from the oyster Crassostrea rhizophorae on bivalve circulating hemocytes. PloS One 8: e57384.

Schenk G, Mitic N, Graeme RH and Comba P (2013). Purple acid phosphatase: A journey into the function and mechanism of a colorful enzyme. Coord. Chem. Rev. 257: 473-482.

Tiscar PG and Mosca F (2004). Defense mechanisms in farmed marine molluscs. Vet. Res. Commun. 28 (Suppl 1): 57-62.

Wang Y, Norgård M and Andersson G (2005). N-glycosylation influences the latency and catalytic properties of mammalian purple acid phosphatase. Arch. Biochem. Biophys. 435: 147-156.

Xiong X, Feng Q, Chen L, Xie L, et al. (2008). Cloning and characterization of an IKK homologue from pearl oyster, Pinctada fucata. Dev. Comp. Immunol. 32: 15-25.

Zhang D, Jiang J, Jiang S, Ma J, et al. (2009a). Molecular characterization and expression analysis of a putative LPSinduced TNF-alpha factor (LITAF) from pearl oyster Pinctada fucata. Fish Shellfish Immunol. 27: 391-396.

Zhang D, Jiang S, Qiu L, Su T, et al. (2009b). Molecular characterization and expression analysis of the IkappaB gene from pearl oyster Pinctada fucata. Fish Shellish Immunol. 26: 84-90.

Zhao X, Wang Q, Jiao Y, Huang R, et al. (2012). Identification of genes potentially related to biomineralization and immunity by transcriptome analysis of pearl sac in pearl oyster Pinctada martensii. Marine Biotechnol. (NY) 14: 730-739. 